\title{
Integral inequalities under beta function and preinvex type functions
}

\author{
Izhar Ahmad*
}

\author{
${ }^{*}$ Correspondence: \\ drizhar@kfupm.edu.sa \\ Department of Mathematics \\ and Statistics, King Fahd \\ University of Petroleum \\ and Minerals, Dhahran 31261, \\ Saudi Arabia
}

\begin{abstract}
In the present paper, the notion of P-preinvex function is introduced and new integral inequalities for this kind of function along with beta function are establised. The work extends the results appeared in the literature.
\end{abstract}

Keywords: Euler beta function, Integral inequality, Holder's inequality, P-preinvex function

Mathematics Subject Classification: 33B15, 26B25, 26D15, 26D10

\section{Background}

Convexity plays an important role in economics, management science, engineering, finanace and optimization theory. Many interesting generalizations and extensions of classical convexity have been used in optimization and mathematical inequalities. Hanson (1981) introduced the concept of invexity. These functions were named invex by Craven (1981) and $\eta$-convex by Kaul and Kaur (1980). Weir and Mond (1988) introduced the concept of preinvex function. Later, Mohan and Neogy (1995) presented few properties of preinvex functions. Some refinements of the mathematical inequalities on convex and generalized convex functions have been investigated in Barani et al. (2012), Chalco-Cano et al. (2012), Dragomir (2001), Dragomir and Agarwal (1998), Fok and Vong (2015), Matloka (2014), Muddassar and Bhatti (2013) and Pachpatte (2004).

Let $S$ be a nonempty subset of $R^{n}$ and let $\eta: S \times S \rightarrow R^{n}$.

Definition 1 The set $S \subseteq R^{n}$ is said to be invex with respect to $\eta(u, v)$ if for every $u, v \in S$ and $t \in[0,1]$,

$$
v+t \eta(u, v) \in S .
$$

It is obvious that every convex set is invex with respect to $\eta(u, v)=u-v$, but there exist invex sets which are not convex (see Mohan and Neogy 1995).

Definition 2 (Weir and Mond 1988) The function $f: S \rightarrow R$ is said to be preinvex on $S$ with respect to $\eta(u, v)$, if 


$$
f(v+t \eta(u, v)) \leq(1-t) f(v)+t f(u)
$$

for every $u, v \in S$ and $t \in[0,1]$,

The Gauss-Jacobi type quadrature formula has the following

$$
\int_{a}^{b}(x-a)^{p}(b-x)^{q} f(x) d x=\sum_{k=0}^{\infty} B_{m, k} f\left(\gamma_{k}\right)+R_{m}^{\star}|f|,
$$

for certain $B_{m, k}, \gamma_{k}$ and rest $R_{m}^{\star}|f|$ (see Stancu et al. 2002).

Recently, Liu (2014) obtained several integral inequalities for the left hand side of (1) under the following $P$-convexity:

The function $f: I \rightarrow R$, where $I \subseteq R$ is said to be $P$-convex on a convex set, if

$$
f(t u+(1-t) v) \leq f(u)+f(v) .
$$

for every $u, v \in I$ and $t \in[0,1]$. For the applications of $P$-convex function and its generalizations, we refer Akdemir and Ozdemir (2010), Barani and Barani (2012), Liu (2013, 2014), Tunc (2013) and Varosanec (2007).

The main purpose of this paper is to introduce the class of $P$-preinvex function and derive new inequalities for the left hand side of (1) under these assumptions. The presented results generalize the results of Liu (2014) and references cited therein.

\section{New integral inequalities}

Definition 3 The function $f: S \rightarrow R$ is said to be $P$-preinvex on $S$ with respect to $\eta(u, v)$, if

$$
f(v+t \eta(u, v)) \leq f(u)+f(v) .
$$

for every $u, v \in S$ and $t \in[0,1]$,

Note that every P-convex function (Liu 2014) is a P-preinvex function with respect to $\eta(u, v)=u-v$ for any $t \in[0,1]$.

Lemma 1 Let $f: S=[a, a+\eta(b, a)] \rightarrow R$ be a continous function on the interval of real numbers $S^{0}$ (the interior of $S$ ) with $a<a+\eta(b, a)$. If $f$ is P-preinvex function on $[a, a+\eta(b, a)]$, then for some fixed $p, q>0$,

$\int_{a}^{a+\eta(b, a)}(x-a)^{p}(a+\eta(b, a)-x)^{q} f(x) d x=\eta(b, a)^{p+q+1} \int_{0}^{1} t^{p}(1-t)^{q} f(a+t \eta(b, a)) d t$.

Proof It is easy to observe that

$$
\begin{aligned}
\int_{a}^{a+\eta(b, a)}(x-a)^{p}(a+\eta(b, a)-x)^{q} f(x) d x= & \int_{0}^{1}(a+t \eta(b, a)-a)^{p}(a+\eta(b, a)-a-t \eta(b, a))^{q} \\
& \times f(a+t \eta(b, a)) d t \\
= & \eta(b, a)^{p+q+1} \int_{0}^{1} t^{p}(1-t)^{q} f(a+t \eta(b, a)) d t
\end{aligned}
$$

The following definition will be used in the sequel. 
Definition 4 The beta function is defined for $x, y>0$ as

$$
\beta(x, y)=\int_{0}^{1} t^{x-1}(1-t)^{y-1} d t .
$$

Theorem 1 Let $f: S=[a, a+\eta(b, a)] \rightarrow R$ be a continous function on the interval of real numbers $S^{0}$ (the interior of $S$ ) with $a<a+\eta(b, a)$. If $|f|$ is P-preinvex function on $[a, a+\eta(b, a)]$, then for some fixed $p, q>0$,

$\int_{a}^{a+\theta(b, a)}(x-a)^{p}(a+\eta(b, a)-x)^{q} f(x) d x \leq \eta(b, a)^{p+q+1} \beta(p+1, q+1)(|f(a)|+|f(b)|)$.

Proof Since $|f|$ is $P$-preinvex function on $[a, a+\eta(b, a)]$, we have

$$
|f(a+t \eta(b, a))| \leq|f(a)|+|f(b)|
$$

for all $t \in[0,1]$. By Theorem 1 and $P$-preinvexity of $|f|$, we get

$$
\begin{aligned}
\int_{a}^{a+\eta(b, a)}(x-a)^{p}(a+\eta(b, a)-x)^{q} f(x) d x & =\eta(b, a)^{p+q+1} \int_{0}^{1} t^{p}(1-t)^{q}|f(a+t \eta(b, a))| d t \\
& \leq \eta(b, a)^{p+q+1} \int_{0}^{1} t^{p}(1-t)^{q}(|f(a)|+|f(b)|) d t \\
& =\eta(b, a)^{p+q+1} \beta(p+1, q+1)(|f(a)|+|f(b)|)
\end{aligned}
$$

(by the definition 4 ).

Theorem 2 Let $f: S=[a, a+\eta(b, a)] \rightarrow R$ be a continous function on the interval of real numbers $S^{0}$ (the interior of $S$ ) with $a<a+\eta(b, a)$. If $|f|^{\frac{k}{k-1}}$ is P-preinvex function on $[a, a+\eta(b, a)]$, then for some fixed $p, q>0$,

$$
\begin{aligned}
& \int_{a}^{a+\eta(b, a)}(x-a)^{p}(a+\eta(b, a)-x)^{q} f(x) d x \\
& \quad \leq \eta(b, a)^{p+q+1}[\beta(k p+1, k q+1)]^{\frac{1}{k}}\left(|f(a)|^{\frac{k}{k-1}}+|f(b)|^{\frac{k}{k-1}}\right)^{\frac{k-1}{k}} .
\end{aligned}
$$

Proof The P-preinvexity of $|f|^{\frac{k}{k-1}}$ on $[a, a+\eta(b, a)]$ along with Lemma 1, Definition 4 and Hölder inequality imply that

$$
\begin{aligned}
& \int_{a}^{a+\eta(b, a)}(x-a)^{p}(a+\eta(b, a)-x)^{q} f(x) d x \\
& \quad \leq \eta(b, a)^{p+q+1}\left[\int_{0}^{1} t^{k p}(1-t)^{k q}\right]^{\frac{1}{k}}\left[\int_{0}^{1}|f(a+t \eta(b, a))|^{\frac{k}{k-1}} d t\right]^{\frac{k-1}{k}} \\
& \quad \leq \eta(b, a)^{p+q+1}[\beta(k p+1, k q+1)]^{\frac{1}{k}}\left[\int_{0}^{1}\left(|f(a)|^{\frac{k}{k-1}}+|f(b)|^{\frac{k}{k-1}}\right) d t\right]^{\frac{k-1}{k}} \\
& \quad=\eta(b, a)^{p+q+1}[\beta(k p+1, k q+1)]^{\frac{1}{k}}\left(|f(a)|^{\frac{k}{k-1}}+|f(b)|^{\frac{k}{k-1}}\right)^{\frac{k-1}{k}} .
\end{aligned}
$$

This completes the proof. 
Theorem 3 Let $f: S=[a, a+\eta(b, a)] \rightarrow R$ be a continous function on the interval of real numbers $S^{0}$ (the interior of $S$ ) with $a<a+\eta(b, a)$. If $|f|^{l}$ is P-preinvex function on $[a, a+\eta(b, a)]$, then for some fixed $p, q>0$,

$$
\int_{a}^{a+\eta(b, a)}(x-a)^{p}(a+\eta(b, a)-x)^{q} f(x) d x \leq \eta(b, a)^{p+q+1} \beta(p+1, q+1)\left(|f(a)|^{l}+|f(b)|^{l}\right) .
$$

Proof The P-preinvexity of $|f|^{l}$ on $[a, a+\eta(b, a)]$ along with Lemma 1, Definition 4 and Hölder inequality give

$$
\begin{aligned}
& \int_{a}^{a+\eta(b, a)}(x-a)^{p}(a+\eta(b, a)-x)^{q} f(x) d x \\
& \quad=\eta(b, a)^{p+q+1} \int_{0}^{1}\left[t^{p}(1-t)^{q}\right]^{\frac{l-1}{l}}\left[t^{p}(1-t)^{q}\right]^{\frac{l}{l}} f(a+t \eta(b, a)) d t \\
& \quad \leq \eta(b, a)^{p+q+1}\left[\int_{0}^{1} t^{p}(1-t)^{q} d t\right]^{\frac{l-1}{l}}\left[\int_{0}^{1} t^{p}(1-t)^{q}|f(a+t \eta(b, a))| d t\right]^{\frac{1}{l}} \\
& \quad \leq \eta(b, a)^{p+q+1}[\beta(p+1, q+1)]^{\frac{1-l}{l}}\left[\left(|f(a)|^{l}+|f(b)|^{l}\right) \beta(p+1, q+1)\right]^{\frac{1}{l}} \\
& \quad=\eta(b, a)^{p+q+1} \beta(p+1, q+1)\left(|f(a)|^{l}+|f(b)|^{l}\right)^{\frac{1}{l}} .
\end{aligned}
$$

This completes the proof.

\section{Intergal inequalities involving prequasi-invex}

I state the following theorems as the proof follow on the same lines of the theorems of "New integral inequalities" section.

Definition 5 (Pinni 1991) The function $f: S \rightarrow R$ is said to be prequasi-invex on $S$ with respect to $\eta(u, v)$, if

$$
f(t u+(1-t) v) \leq \max (f(u), f(v))
$$

for every $u, v \in S$ and $t \in[0,1]$.

Theorem 4 Let $f: S=[a, a+\eta(b, a)] \rightarrow R$ be a continous function on the interval of real numbers $S^{0}$ (the interior of $S$ ) with $a<a+\eta(b, a)$. If $f$ is prequasi-invex function on $[a, a+\eta(b, a)]$, then for some fixed $p, q>0$

$\int_{a}^{a+\eta(b, a)}(x-a)^{p}(a+\eta(b, a)-x)^{q} f(x) d x \leq \eta(b, a)^{p+q+1} \beta(p+1, q+1) \max (f(a), f(b))$.

Theorem 5 Let $f: S=[a, a+\eta(b, a)] \rightarrow R$ be a continous function on the interval of real numbers $S^{0}$ (the interior of $S$ ) with $a<a+\eta(b, a)$. If $|f|$ is prequasi-invex function on $[a, a+\eta(b, a)]$, then for some fixed $p, q>0$, 
$\int_{a}^{a+\eta(b, a)}(x-a)^{p}(a+\eta(b, a)-x)^{q} f(x) d x \leq \eta(b, a)^{p+q+1} \beta(p+1, q+1) \max (|f(a)|,|f(b)|)$.

Theorem 6 Let $f: S=[a, a+\eta(b, a)] \rightarrow R$ be a continous function on the interval of real numbers $S^{0}$ (the interior of $S$ ) with $a<a+\eta(b, a)$. If $|f|^{\frac{k}{k-1}}$ is prequasi-invex function on $[a, a+\eta(b, a)]$, then for some fixed $p, q>0$,

$$
\begin{aligned}
& \int_{a}^{a+\eta(b, a)}(x-a)^{p}(a+\eta(b, a)-x)^{q} f(x) d x \\
& \quad \leq \eta(b, a)^{p+q+1}[\beta(k p+1, k q+1)]^{\frac{1}{k}} \max \left(|f(a)|^{\frac{k}{k-1}},|f(b)|^{\frac{k}{k-1}}\right)^{\frac{k-1}{k}} .
\end{aligned}
$$

Theorem 7 Let $f: S=[a, a+\eta(b, a)] \rightarrow R$ be a continous function on the interval of real numbers $S^{0}$ (the interior of $S$ ) with $a<a+\eta(b, a)$. If $|f|^{l}$ is prequasi-invex function on $[a, a+\eta(b, a)]$, then for some fixed $p, q>0$,

$\int_{a}^{a+\eta(b, a)}(x-a)^{p}(a+\eta(b, a)-x)^{q} f(x) d x \leq \eta(b, a)^{p+q+1} \beta(p+1, q+1) \max \left(|f(a)|^{l},|f(b)|^{l}\right)$.

Remark 1 If $\eta(b, a)=b-a$ in the theorems of "Intergal inequalities involving prequasi-invex" section, then we get the Theorems appeared in Liu (2013).

\section{Conclusion}

In this paper, I have introduced the P-preinvex function and used it along with beta function to establish the new integral type inequalities. I also stated the other integral type inequalities under prequasi-invex function. The presented results may be futher generalized under weaker convexity assumptions.

\section{Acknowledgements}

This research is supported by King Fahd University of Petroleum and Minerals, Saudi Arabia under the Internal Project No. IN131038. The author is thankful to the anonymous referees for their valuable suggestions, which have substantially improved the presentation of the paper.

\section{Competing interests}

The author declares that he has no competing interests.

Received: 12 December 2015 Accepted: 15 April 2016

Published online: 26 April 2016

\section{References}

Akdemir AO, Ozdemir ME (2010) Some Hadamard-type inequalities for coordinated P-convex functions and GodunovaLevin functions. AIP Conf Proc 139:7-15

Barani A, Barani S (2012) Hermite-Hadamard type inequalities for functions when a power of the absolute value of the first derivative is P-convex. Bull Austral Math Soc 86:126-134

Barani A, Ghazanfari AG, Dragomir SS (2012) Hermite-Hadamard inequality for functions whose derivatives absolute values are preinvex. J Inequal Appl 2012:47

Craven BD (1981) Invex functions and constrained local minima. Bull Aust Math Soc 24:357-366

Chalco-Cano Y, flores-Franulic A, Roman-Flores H (2012) Ostrowski type inequalities for interval-valued functions using generalized Hukuhara derivative. Comput Appl Math 31:457-472

Dragomir SS (2001) Refinements of the Hermite-Hadamard integral inequality for log-convex function. Aust Math Soc Gaz 28:129-134 
Dragomir SS, Agarwal RP (1998) Two inequalities for differentiable mapping and applications to special mean of real numbers and to trapezoidal formula. Appl Math Lett 11:91-95

Fok H, Vong S (2015) Generalizations of some Hermite-Hadamard-type inequalities. Indian J Pure Appl Math 46:359-370 Kaul RN, Kaur S (1980) Optimality criteria in nonlinear programming involving nonconvex functions. J Math Anal Appl 105:104-112

Hanson MA (1981) On sufficiency of the Kuhn-Tucker conditions. J Math Anal Appl 80:545-550

Liu W (2013) New integral inequalities via $(\alpha, m)$-convexity and quasi-convexity. Hacet J Math 42:289-297

Liu W (2014) Some Simpson type inequalities for h-convex and $(\alpha, m)$-convex functions. J Comput Anal Appl 16:1005-1012

Liu W (2014) New integral inequalities involving beta function via P-convexity. Miskolc Math Notes 15:585-591

Matloka M (2014) Inequalities for h-preinvexs. Appl Math Comput 234:52-57

Mohan SR, Neogy SK (1995) On invex sets and preinvex functions. J Math Anal Appl 189:901-908

Muddassar M, Bhatti MI (2013) Some generalizations of Hadamard's-type inequalities through differentiability for s-convex functions. Indian J Pure Appl Math 44:131-151

Pachpatte BG (2004) A note on integral inequalities involving two log-convex functions. Math Inequal Appl 7:511-515

Pinni R (1991) Invexity and generalized convexity. Optimization 22:513-525

Stancu DD, Coman G, Blaga P (2002) Analiza Numerica si teroia approximarii, vol II. Presa Universitara Clujeana, Cluj-Napoca

Tunc M (2013) On some integral inequalities via h-convexity. Moskolc Math Notes 14:1041-1057

Varosanec S (2007) On h-convexity. J Math Anal Appl 326:303-311

Weir A, Mond B (1988) Preinvex functions in multiple objective optimization. J Math Anal Appl 136:29-38

\section{Submit your manuscript to a SpringerOpen ${ }^{\circ}$ journal and benefit from:}

- Convenient online submission

\section{- Rigorous peer review}

- Immediate publication on acceptance

- Open access: articles freely available online

- High visibility within the field

Retaining the copyright to your article

Submit your next manuscript at $\boldsymbol{\nabla}$ springeropen.com 